Original scientific paper

\title{
FORMATION MECHANISM OF AN ADHERENT VORTEX IN THE SIDE PUMP SUMP OF A PUMPING STATION
}

\author{
Xi, W. \& Lu, W. G." \\ College of Hydraulic Science and Engineering, Yangzhou University, Yangzhou, 225009 China \\ E-Mail: wglu@yzu.edu.cn ( ${ }^{\#}$ Corresponding author)
}

\begin{abstract}
The inflow of the side pump sump is not smooth enough during the operation of a pumping station, resulting in an asymmetric adherent vortex that endangers the station's normal operation and safety. To solve this problem, flow field and vorticity distribution charts of different flow layers were created through the establishment of a numerical model of the pumping station inflow by means of the fluid simulation software. The formation mechanism of the asymmetric adherent vortex in the side pump sump was analysed by combining Reynolds shear stress distribution, the simplified Navier-Stokes equation, and the transport equation of turbulent kinetic energy. Furthermore, the accuracy of the numerical simulation results was verified using flow field data collected via particle image velocimetry at the junction of the forebay and pump sumps of the station. Results show that the distribution of inflow velocity is uneven due to the asymmetric friction of the inflow in the side pump sump. The transverse velocity formed from it generates the asymmetric vortex in the side pump sump and creates an inspiratory vortex.

(Received in February 2021, accepted in April 2021. This paper was with the authors 1 month for 2 revisions.)
\end{abstract}

Key Words: Pump Station Engineering, Side Pump Sump, Adherent Vortex

\section{INTRODUCTION}

Pump station engineering plays an important role in the allocation of water resources, urban flood control, obtainment of high and stable crop yield, and good harvest during droughts and floods. Large and medium-sized pumping stations generally comprise multiple units that have corresponding multiple pump sumps. The upstream of the pump sumps has a forebay and a diversion channel, whose function is to guide the water flow to the pump sump. However, in the actual operation of a multi-unit pumping station, the inflow of the side unit is not smooth, causing transverse velocity and vortexing that lead to a low flow rate in the side pump sump. This phenomenon results in the vibration of units and affects the working efficiency of the sump and the safety of the entire pumping station.

Numerous large and medium-sized pumping stations have been built in response to the development of societies and the increasing demand for water resource allocation. The complex flow pattern of the pump sump cannot meet the requirements of safe operation of pumping stations. Hence, analysing the cause of the vortex in the pump sump of a pumping station is important. Many fluid simulation software programs have been proposed. In terms of numerical simulation theory [1-6] research, with turbulence model analysis and the turbulent kinetic energy equation having been put forward $[7,8]$, an increasing number of high-precision numerical simulation techniques are used to simulate the complex flow in the side pump sump of a pumping station. The 3D unsteady turbulent flow in the sump has been simulated with four different turbulence models, namely, standard $k-\varepsilon$, realizable $k-\varepsilon$, renormalization group $k-\varepsilon$, and shear stress transfer $k-\varepsilon$ volume of fluid (VOF) multiphase models [9]. The causes and influencing factors of the inspiratory vortex in the sump have also been analysed with large eddy simulation (LES) technology [10]. In these previous studies, numerical simulation was used for a detailed analysis, but no physical model was adopted to verify the results, leading to the lack of credibility. In terms of the physical model test, the volumetric three-component velocimetry system [11] has been used to study the variation in 
the velocity gradient, vortex intensity, and kinetic energy in the vortex zone during the process. A study also utilized a miniature physical model of equal ratio [12] in the design optimization of the pump sump. However, these studies merely used physical model tests to study individual engineering, and their results are not universal. In addition, studies on vortex elimination measures in the pump sump have shown that although the anti-vortex device $[13,14]$ is worthy of attention, this technology still lacks maturity and has certain limitations; hence, it cannot be widely applied in pump station engineering.

Given such background, most researchers have conducted optimization research on the improvement of the flow pattern in the pump sump through numerical simulations or physical tests, and only a few of them combined numerical simulation, theoretical analysis, and experimental verification. Similarly, only a few have studied the distribution and formation mechanism of the asymmetric adherent vortex in the side pump sump. In the current study, the distribution of the asymmetric adherent vortex of the side pump sump of a pumping station was summarized through numerical simulation. The causes of the vortex were analysed by combining relevant theories. The formation mechanism was verified using data collected via particle image velocimetry (PIV). The results can provide theoretical support for the optimization of the flow pattern in the side pump sump of pump stations.

\section{STATE OF THE ART}

Several scholars have conducted relevant research mainly in the following aspects. In their research on wall turbulence, Hoyas and Jimenez $[15,16]$ performed many numerical simulations and theoretical demonstrations in which the Reynolds number was in the range of 180-2000 to analyse wall turbulent motion. However, they only applied numerical simulation to study the flow in the horizontal direction and did not verify the physical model test. Adrian [17] studied the structure of hairpin vortex in wall turbulence and analysed its formation through numerical simulation and physical model research. Dixit and Ramesh [18] analysed the pressure gradient of the wall turbulence boundary layer at the confluence interface. They identified and studied the anomalies of logarithmic law but did not analyse the flow velocity distribution in detail. Abreu et al. [19] used direct numerical simulation to analyse turbulent channel flow and obtained its turbulent distribution law through the spectral inherent orthogonal decomposition method and a solvent. With regard to research on the flow around problem, Albers et al. [20] used the LES model and analysed the flow around the wall of a straight plate and the influence of the transverse surface wave of wingspan propagation in the turbulent boundary layer under zero pressure gradient. Many scholars have combined two methods to verify each of them [21], and some of them introduce machine learning method [22], but only a few used a method that combines physical model testing and theoretical analysis [23].

The above-mentioned studies focused on channel flow, wall turbulence, and flow around, and studies on the vortex in the pump sump of a pumping station mostly concentrated on the inspiratory vortex at the bell mouth. Meanwhile, research on the near-wall vortex in the pump sump, especially on the formation mechanism of the asymmetric adherent vortex in the pump sump of pump stations, is scarce. In the current study, numerical simulation was combined with theoretical analysis and experimental verification to analyse the formation mechanism of the asymmetric adherent vortex in the side pump sump of a pump station. The results can provide theoretical guidance for solving the adherent vortex problem.

The rest of this paper is organized as follows. The third section describes the modelling of the inflow in a pumping station and the establishment and test process of the PIV model. In the fourth section, the distribution of the asymmetric adherent vortex of the side pump sump of the pumping station is summarized through numerical simulation. The formation 
mechanism of the asymmetric adherent vortex in the side pump sump is also analysed by combining Reynolds shear stress distribution, the simplified Navier-Stokes (N-S) equation, and the transport equation of turbulent kinetic energy. The last section summarizes the study and provides relevant conclusions.

\section{METHODOLOGY}

\subsection{Construction of a numerical model}

Modelling software was used to build the physical models. Time-averaged equations of LES were selected for the numerical calculation of closed two-phase flow in a large eddy model. The semi-implicit method for pressure-linked equations (SIMPLE) was adopted to solve coupled equations of velocity and pressure. The free water surface was simulated using the VOF method.

The 3D turbulent N-S equation was filtered using a spatial filter function. The governing equation of the large eddy model is:

$$
\frac{\partial \rho}{\partial t}+\frac{\partial \rho u_{i}}{\partial x_{i}}=0
$$

The Reynolds equation is:

$$
\frac{\partial\left(\rho u_{i}\right)}{\partial t}+\frac{\partial\left(\rho u_{i} \rho u_{j}\right)}{\partial x_{j}}=-\frac{\partial p}{\partial x_{j}}+\frac{\partial}{\partial x_{j}}\left(\mu \frac{\partial u_{i}}{\partial x_{j}}\right)+S_{i}
$$

where $x_{i}$ and $x_{j}(i, j=1,2,3)$ are Cartesian coordinates and $\bar{u}_{i}, \bar{u}_{j}(i, j=1,2,3)$ are the velocity components after filtering. $\bar{p}$ is the pressure after filtering. $g_{i}$ represents the mass force component, and $v$ represents the molecular viscosity coefficient. $\tau_{i j}$ is the sublattice Reynolds stress induced by nonlinear convection in filtering, and $\overline{\tau_{i j}}=\overline{u_{i} u_{j}}-\overline{u_{i}} \overline{u_{j}}$.

Simulation by building a model is necessary. The import of the diversion channel was set as the import boundary of the computational domain. Medium turbulence intensity was set as $T_{u}=5 \%$. The imports of three pumping units were set as the export boundary of the entire calculation domain. Symmetrical boundary treatment was adopted because the water level did not change much, and the shear stress effect of air on the water surface was ignored. The solid wall in the flow region was constrained by the wall boundary condition, and the solid wall was constrained by the non-slip boundary condition. Fluid was static at the solid wall. The second-order upwind scheme with good adaptability that considers the effect of gravity in the calculation process was utilized, and the PISO algorithm was adopted for unsteady compressible flow calculation. Except for the inlet, outlet, and free water surface, the rest were solid walls. The number of calculation steps was 2000 , and the convergence accuracy was $10^{-7}$.

Unstructured grid partition was used for the local encryption of the entire computational domain due to the complex structure. In the range of the side pump sump (Fig. 1), hydraulic loss was adopted as an appropriate parameter to measure the number of grids. When the number of grids reached 4,304,570, the hydraulic loss was unchanged, and the difference did not exceed $2 \%$.

\subsection{Construction of the PIV test device}

A test device was designed in combination with the three-unit pumping station in an actual project to verify and analyse the numerical simulation results. The test device is shown in Fig. 2. For the test, data on flow field characteristics were collected via PIV. This technology can obtain the entire information of the liquid flow in the shooting field of view and extract 
the real-time data needed for analysis through relevant software. The test was conducted under constant flow conditions, and the test temperature was $200{ }^{\circ} \mathrm{C}$. Before the test, the equipment was installed and connected, and the chip light source produced by laser at the testing section was aligned. The CCD camera's position was adjusted, and the corresponding test parameters of PIV were set. The particle image of the testing section was clearly presented on a computer screen, and the camera and laser light source were not shaken nor displaced during the test. During the test, the circulating water pump was turned on to form a stable water circulation, the flow through the valve on the water supply pipe was adjusted, and the water level in the testing section was set and made stable. After the water flow passed through the test model section, it flowed into the lower circulating water tank. The water flow circulated in the test device. After the water flow in the testing section became stable, the PIV system was used to collect the flow field corresponding to the research position of the forebay and pump sump. The specific test arrangement is shown in Fig. 2.

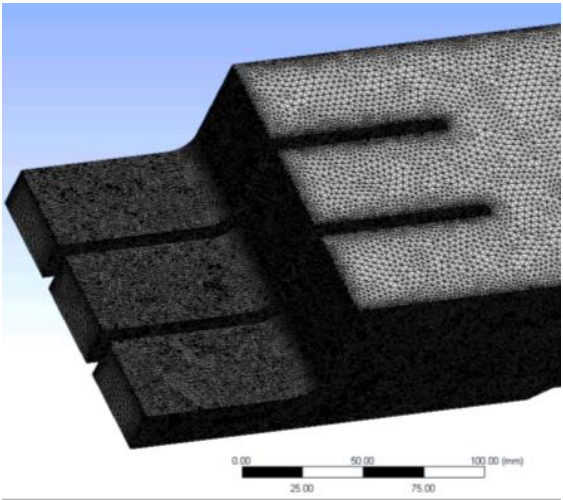

Figure 1: Grid division diagram of local encryption.

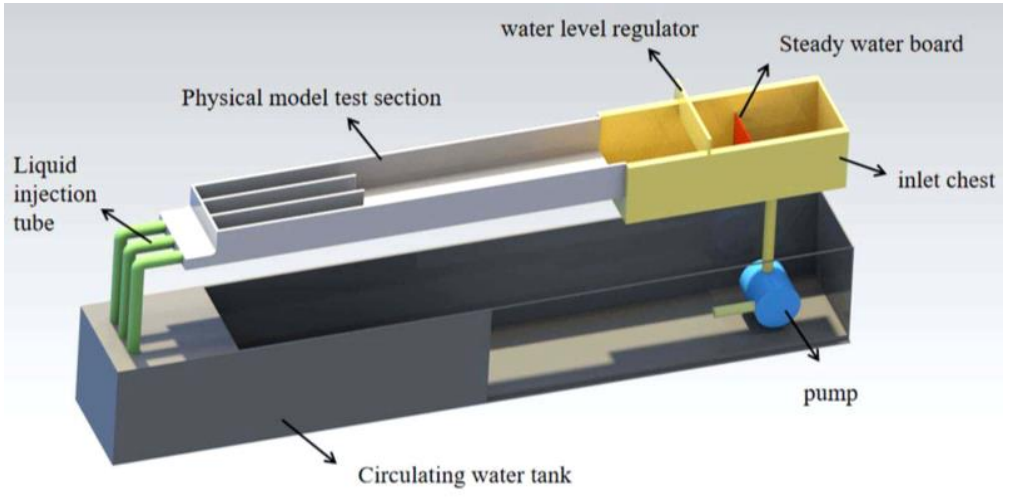

Figure 2: Diagram of the PIV test device.

\section{SIMULATION ANALYSIS AND DISCUSSION}

\subsection{Analysis of numerical simulation}

Analysis of 3D flow characteristics: The numerical simulation method was used to analyse the characteristics of the water flow in the side and non-side pump sumps. The streamline distribution of the side and non-side pump sumps is shown in Fig. 3. In the non-side pump sump, the water flow was acted on by the pump, and the direction of the flow line did not change significantly along the flow on the horizontal plane. In the vertical axis, the water flowed to the bottom of the downstream water inlet to bend, the transition was smooth, and the streamline was straight. In the side pump sump, the streamlines bended near the side wall, and many low-velocity areas were created near the wall to form a small vortex band. The flow velocity on both sides of the forebay was asymmetrical, and the streamline was deflected at the front edge of the isolated pier. Rear transverse velocity flow occurred and resulted in a disordered curved line. The upper flow line affected the bend after entering the side of the pump sump, and it showed a tendency to form an irregular vortex. At the same time, the transition of the streamline into the pump sump was not smooth, and the flow state in front of the water inlet was turbulent.

In the pumping station, the inflow of the non-side pump sump was smooth and straight, and the flow pattern was good. By contrast, the streamline of the side pump sump was scattered, and many vortices attached to the wall, which affected the pump inlet. 


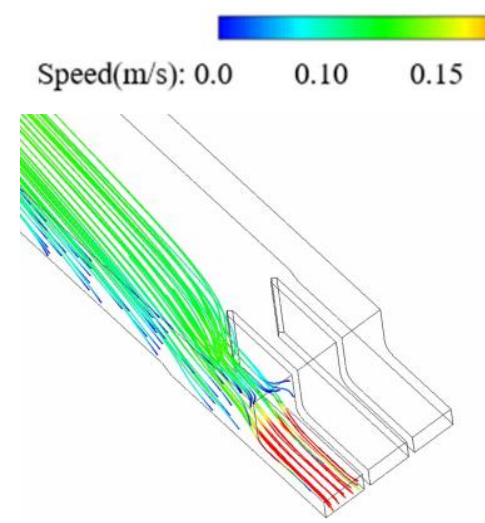

a)

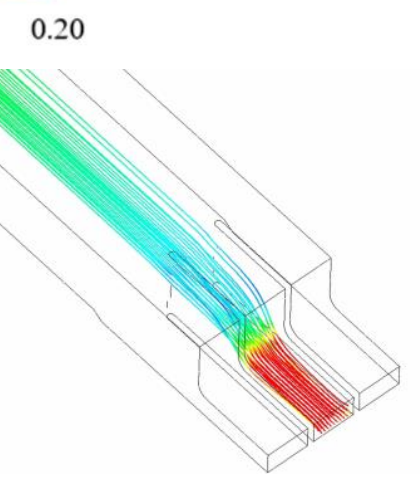

b)

Figure 3: Schematic of the numerical simulation flow field; a) streamline figure of the non-side pump sump, b) streamline figure of the side pump sump.

Analysis of 2D horizontal flow field: Flow field velocity cloud figures of the five flow layers (Fig. 4) were obtained in accordance with the numerical simulation results. Fig. 4 shows that the flow velocity near the isolated pier of the side pump sump was relatively high, and the velocity near the side wall attenuated sharply along the flow direction. The flow velocity direction of the entire side pump sump was inclined to the intermediate isolated pier, and the downstream side of the side pump sump tended to produce low-speed adherent vortices on the side wall.

The analysis above indicates that the water flowed through flow layer a down to flow layer e, and the inflow in the side pump sump generally began from the inlet and flowed toward the isolated pier obliquely. An adherent vortex was generated at the downstream side wall. This vortex is caused by the asymmetry of the force on both sides of the water flow when the water entered. The frictional resistance between the inflow water body and the solid side wall near the side wall was greater than the shear stress between the water flow and the water flow in front of the isolated pier. This phenomenon can be analysed with Reynolds shear stress distribution.

In actual engineering, the diversion channel of pumping station projects often cannot meet the requirements in terms of length, and it is likely to cause instability of the water flow in the pump sump and the phenomenon of vortexing. In the flat flow of the forebay and pump sump, a linear relationship exists between the total average shear stress and the distance to the wall. The maximum value occurs at the wall, and the minimum value is at the centre point. The greater the Reynolds number is, the closer the Reynolds shear stress curve is to the total average shear stress curve. This condition indicates that the Reynolds shear stress near the wall of the pump sump increases, and the stress increases with the increase in the Reynolds number. In pump station engineering, the water flow is turbulent, and the Reynolds number is large. Thus, considerable shear stress is generated near the wall, which then forms large velocity and pressure gradients. These gradients cause the strain rate tensor to increase, which increases the vorticity and produces a vortex.

In this study, the Reynolds shear stress near the wall was large, the flow velocity gradient was large, and the flow velocity near the wall was low. At the isolated pier, the Reynolds shear stress on the front edge of the isolated pier was large, the boundary layer was thick, and no turbulence generation and diffusion occurred. The turbulent dissipation term was offset by the viscous diffusion term. In the downstream flow, the boundary layer of the isolated pier became thin, and the turbulence gradually diffused. The pressure and velocity gradients gradually exchanged positive and negative values, and turbulence was slowly generated. The transport of turbulent terms occurred between the side wall and the isolated pier, causing the water flow to shift laterally. A vortex formed easily near the wall downstream of the side wall 
where the boundary layer was relatively stable. When these data were combined with the data observed in the experiment, we found that due to the asymmetric inflow conditions, a largescale vortex was prone to occur near the wall downstream of the inner wall of the side pump sump.

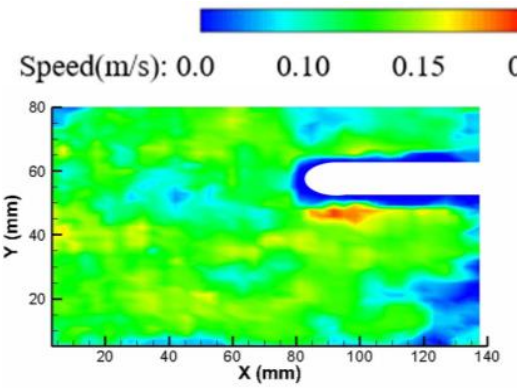

Section a

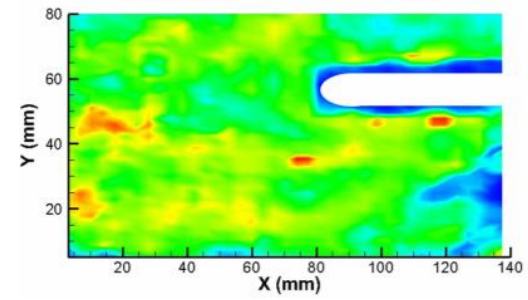

Section d

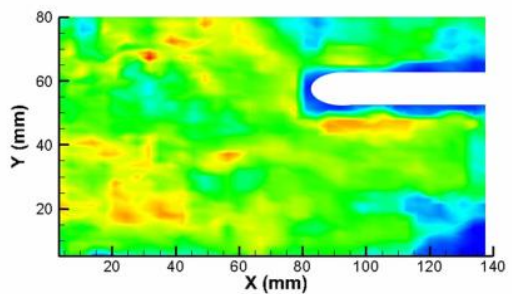

Section $b$

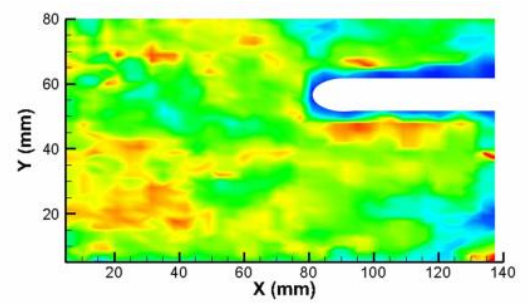

Section e

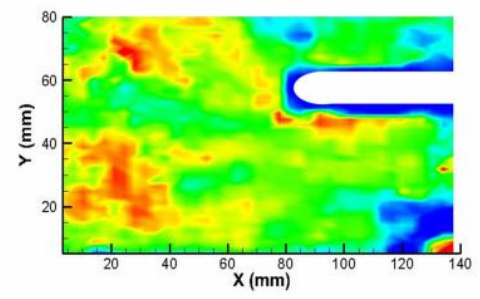

Section c

Figure 4: Flow velocity nephogram of the horizontal plane based on numerical methods.

Analysis of the hydraulic characteristics of the sections: The flow field in the side pump sump was analysed using a software. The horizontal flow field of different water depths was selected. The specific section distribution map is shown in Fig. 5. The flow velocity perpendicular to the flow direction was extracted for analysis, and the vorticity field was studied to explore the changes in liquid clusters.

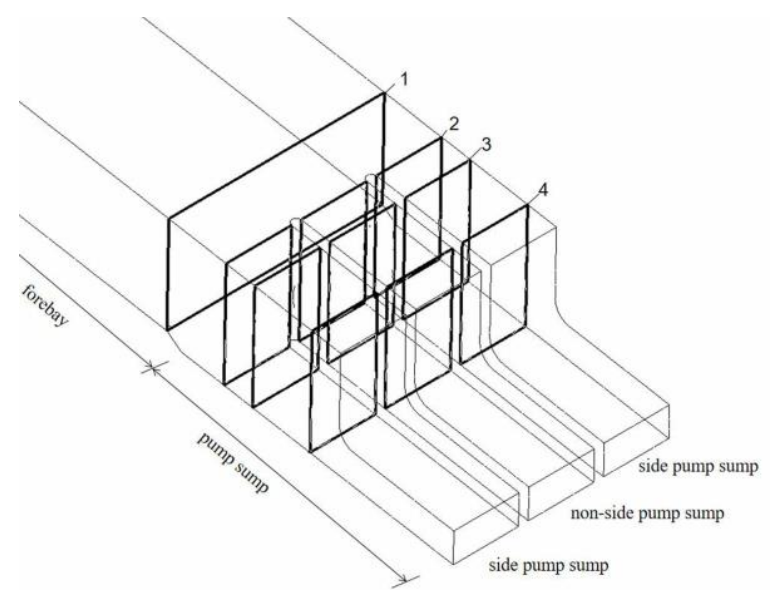

Figure 5: Schematic of section location.

Fig. 6 shows a cross-sectional flow velocity distribution diagram of different positions of the side pump sump (the left coordinate axis is the position of the isolated pier, and the position of $x=60 \mathrm{~mm}$ is the side wall). In Section 1 in front of the isolated pier at different water depths, the flow velocity distribution had no obvious change in the vertical direction. The flow velocity was low near the wall on both sides, the flow velocity was high in the middle, and the peak and valley distributions were similar. In Section 2 of the isolated pier, the flow velocity changed significantly in the vertical direction, and with the increase in water depth, the amplitude of flow velocity fluctuation, the number of peaks and valleys, and the 
flow fluctuation at the bottom increased. In Section 3 behind the isolated pier, the flow velocity fluctuation began to slow down, but the flow velocity near the side wall began to decrease sharply. The flow velocity at the side wall declined obviously with the increase in water depth. When the water flow completely entered the side pump sump, the flow velocity distribution in Section 4 did not change significantly in the vertical direction; the flow velocity near the side wall continued to decrease, and the water flow at the side wall fluctuated significantly.

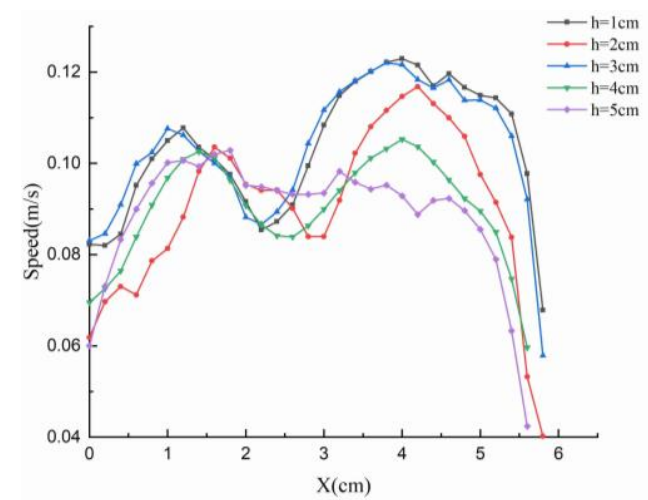

Section 1

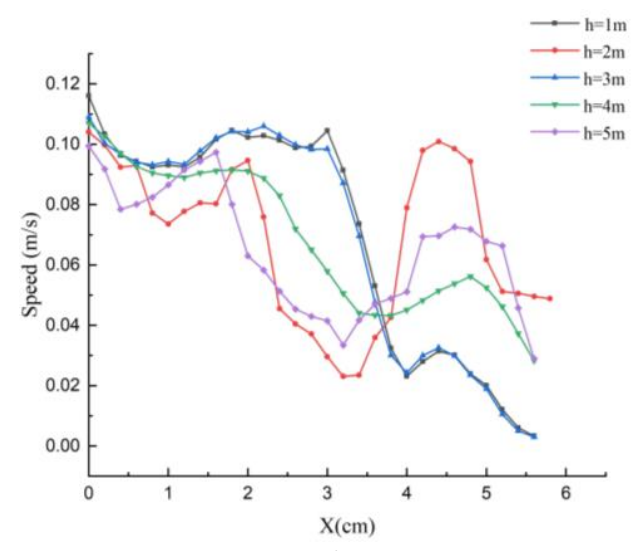

Section 3

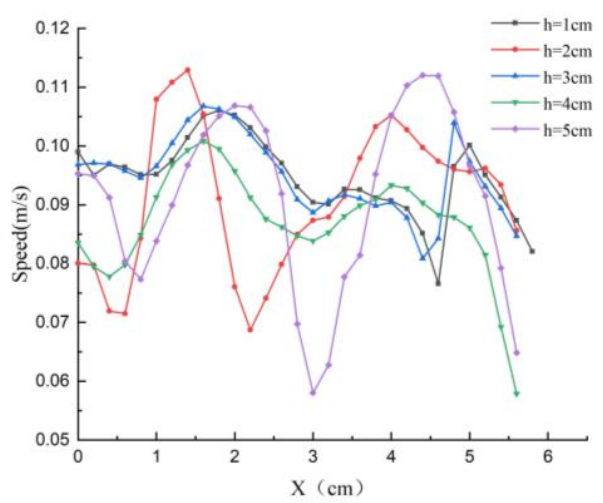

Section 2

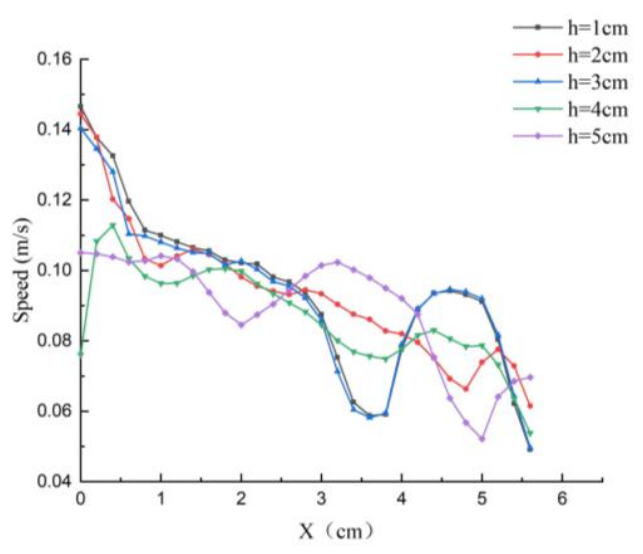

Section 4

Figure 6: Schematic of the cross-sectional flow rate.

Fig. 7 shows a cross-sectional vorticity distribution diagram of different positions of the side pump sump (the left coordinate axis is the position of the isolated pier, and the position of $x=60 \mathrm{~mm}$ is the side wall). The figure shows that the vorticity distribution in Section 1 in front of the isolated pier at different water depths was relatively stable in the vertical direction. The vorticity near the side wall was large, and the vorticity near the isolated pier was small. In Section 2 of the isolated pier, the vorticity fluctuated significantly in the vertical direction, and as the water depth increased, the vorticity fluctuation intensified. In Section 3 behind the isolated pier, the vorticity fluctuation position moved to the side wall, and the fluctuation gradually decreased as the water depth increased. When the water flow completely entered the pump sump, the surface vorticity in Section 4 fluctuated sharply. As the water depth increased, the fluctuations gradually decreased and spread from the middle to the two side walls.

Analysis of Fig. 7 indicates that the flow micromasses in the side pump sump were relatively stable. The flow micromasses close to the side wall had strong rotation due to the effect of the side wall. When they passed through the isolated pier, the flow micromasses were affected by the front edge of the isolated pier and produced strong rotation. After entering the side pump sump, the flow micromasses began to swirl vigorously, forming a vertical spiral flow and creating a vortex. 


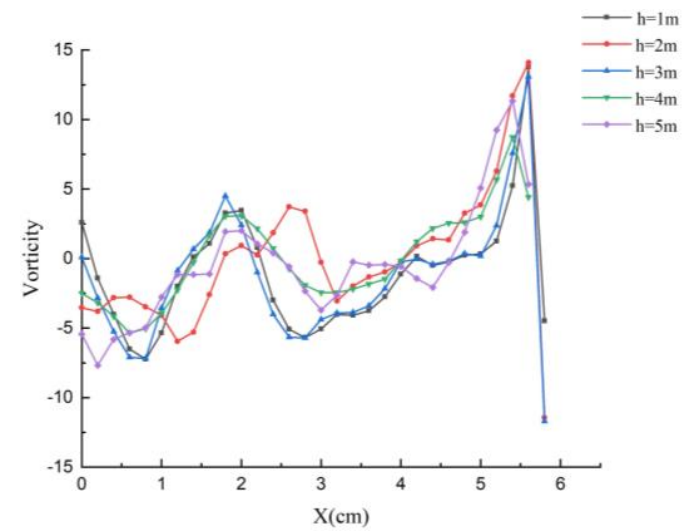

Section 1

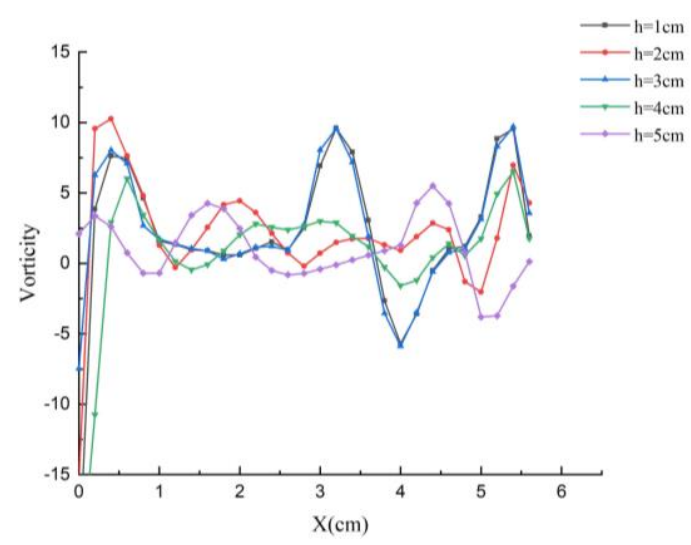

Section 3

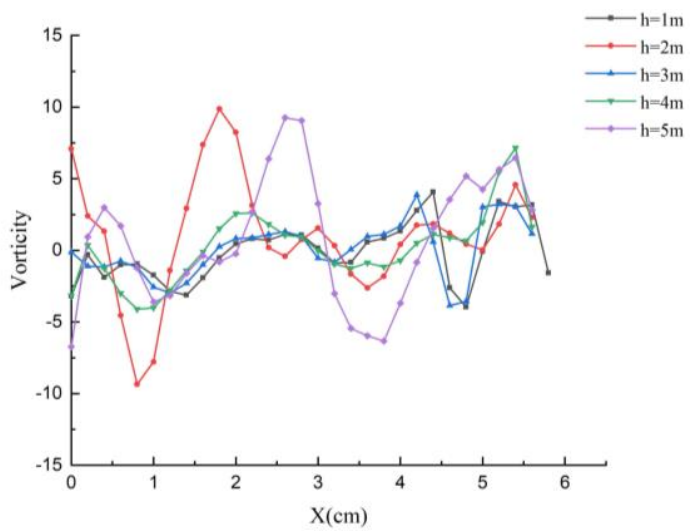

Section 2

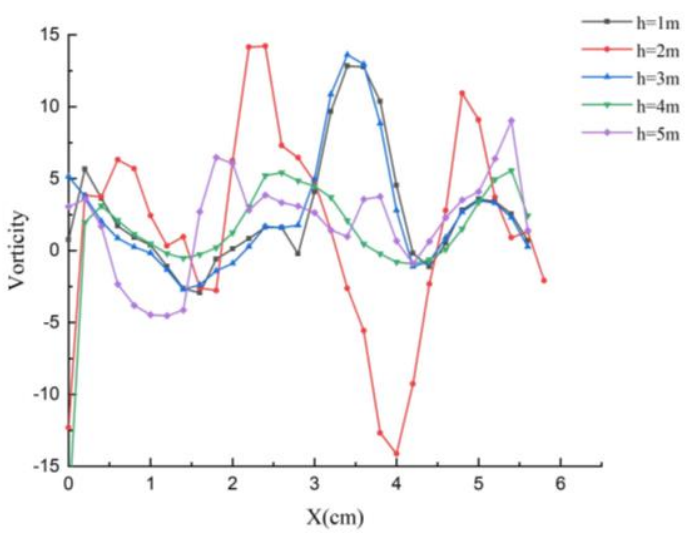

Section 4

Figure 7: Schematic of cross-sectional vorticity.

Theoretical analysis of flow characteristics: The results of the numerical simulation test revealed that the difference in flow velocity on both sides of the inflow of the forebay was extremely large that it caused an uneven flow velocity distribution, resulting in a significant deflection of the flow direction of the side pump sump. Moreover, a large adherent vortex was produced. The N-S equation was used to derive the average flow energy balance equation, and from this, the turbulent flow energy equation was obtained. Then, the adherent vortex of the side pump sump was analysed.

From the N-S and mass conservation equations and in accordance with the uniform flow incompressible condition, the balance condition of turbulent kinetic energy was determined as follows:

$$
\begin{aligned}
& \frac{\partial}{\partial t}\left(\frac{\rho \overline{U_{i}^{2}}}{2}\right)+\frac{\partial}{\partial x_{i}}\left(\overline{U_{j}} \frac{\rho \overline{U_{i}^{2}}}{2}\right)=\rho \overline{u_{i}^{\prime} u_{j}^{\prime}} \frac{\partial \overline{U_{i}}}{\partial x_{j}}-\overline{\tau_{i j}} \frac{\partial \overline{U_{i}}}{\partial x_{j}}-\frac{\partial\left(\overline{U_{i} P}\right)}{\partial x_{i}}+\frac{\partial}{\partial x_{j}}\left(\overline{U_{i} \tau_{i j}}\right)-\frac{\partial}{\partial x_{i}}\left(\overline{U_{j}} \rho \overline{u_{i}^{\prime} u_{j}^{\prime}}\right) \\
& \frac{\partial\left(\rho \overline{u_{i}^{\prime} u_{j}^{\prime}}\right)}{\partial t}+\frac{\partial}{\partial x_{k}}\left(\rho \overline{u_{i}^{\prime} u_{j}^{\prime} U_{k}}\right)=-\left(\rho \overline{u_{j}^{\prime} u_{k}^{\prime}} \frac{\partial \overline{U_{i}}}{\partial x_{k}}+\rho \overline{u_{i}^{\prime} u_{k}^{\prime}} \frac{\partial \overline{U_{j}}}{\partial x_{k}}\right)-\frac{\partial}{\partial x_{k}}\left(\rho \overline{u_{i}^{\prime} u_{j}^{\prime} u_{k}^{\prime}}\right)-\left(\overline{u_{j}^{\prime} \frac{\partial P^{\prime}}{\partial x_{i}}}+\overline{u_{i}^{\prime} \frac{\partial P^{\prime}}{\partial x_{j}}}\right) \\
& +\frac{\partial}{\partial x_{k}}\left(\overline{u_{j}^{\prime} \tau_{i k}^{\prime}}+\overline{u_{i}^{\prime} \tau_{j k}^{\prime}}\right)-\left(\overline{\tau_{i k}^{\prime} \frac{\partial u_{j}^{\prime}}{\partial x_{k}}}+\overline{\tau_{j k}^{\prime} \frac{\partial u_{i}^{\prime}}{\partial x_{k}}}\right)
\end{aligned}
$$

where $\rho$ represents the density of water; $t$ denotes time; $U_{i}, U_{j}, U_{k}$ are the average speeds in $i, j$, and $k$ directions, respectively; $u_{i}, u_{j}, u_{k}$ are the velocity vectors in $i, j$, and $k$ directions, and $u_{i}^{\prime}, u_{j}^{\prime}, u_{k}^{\prime}$ are the root mean squares of homogeneous turbulent velocity fluctuations in turbulent dissipation terms in $i, j$, and $k$ directions. With the N-S equation and the kinetic energy conservation equation and from the uniform flow incompressible condition, we obtain Eq. (3). 
The turbulent kinetic energy transport equation was analysed with the numerical simulation method. Fig. 8 shows the root mean squares of homogeneous turbulent velocity fluctuations in turbulent dissipation terms away from the wall at different distances.

For $u_{1}$, the turbulent flow field was along the direction of water flow. No turbulent flow production and diffusion occurred at the wall position, and the related quantity of velocity and pressure gradients became 0 . In the process of approaching the wall, the turbulent dissipation term gradually cancelled out the viscous diffusion term, and the turbulent dissipation continued to decrease and reached the maximum negative value on the wall. At a distance of about $1 / 8$ of the width of the pump sump from the side wall, the turbulence production term increased sharply and reached the maximum value. At the centre of the river channel, the turbulence products, diffuses, dissipates, viscous diffusion, and the velocity and pressure gradient gradually increased, approached 0 , and changed smoothly This result shows that the turbulence production term to reach the maximum at a distance of about $1 / 8$ times the width of the pump sump from the side wall, and the turbulent diffusion fluctuated significantly at this position. The velocity component in the $x_{1}$ direction reached the maximum at this position.

For $u_{2}$, this result shows that the horizontal water flow fluctuated obviously from the side wall. Compared with the $x_{1}$ direction, more turbulence occurred at this position. The transverse and longitudinal turbulences were interlaced and affected each other. Vortices were easily produced under the action of viscous friction and baroclinicity of the fluid.

In pump station engineering, the water flow enters the pump sump through the forebay, but the flow patterns of the non-side and side pump sumps are different due to the inlet boundary conditions. PIV technology was used to measure the built physical model in order to further analyse the numerical simulation results, and the horizontal flow layer and vertical axis flow field in the non-side and side pump sumps were studied.

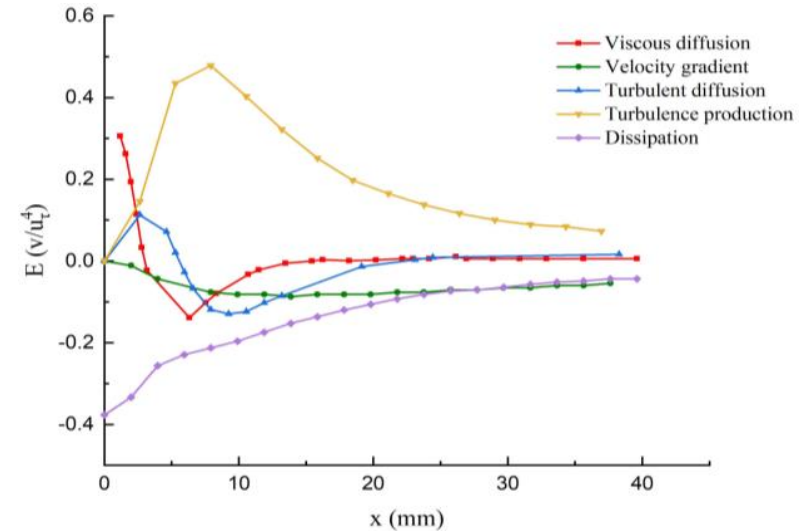

$x_{1}$ direction

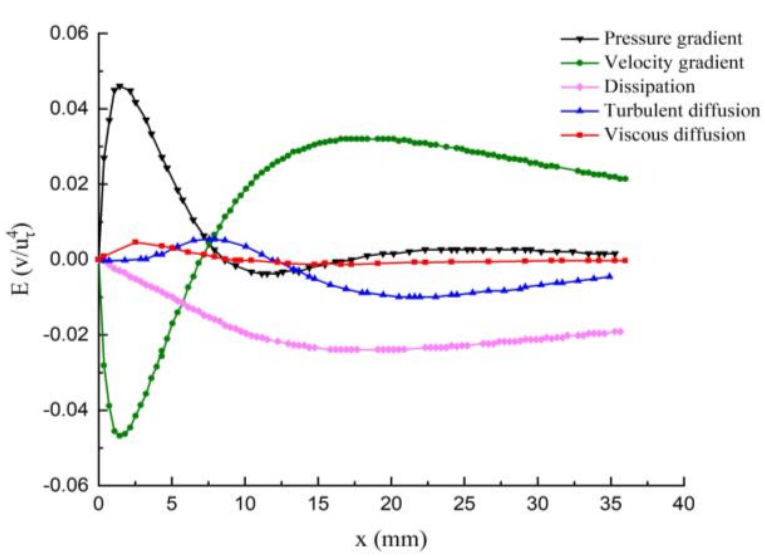

$x_{2}$ direction

Figure 8: Turbulent kinetic energy distribution chart.

\subsection{PIV test verification}

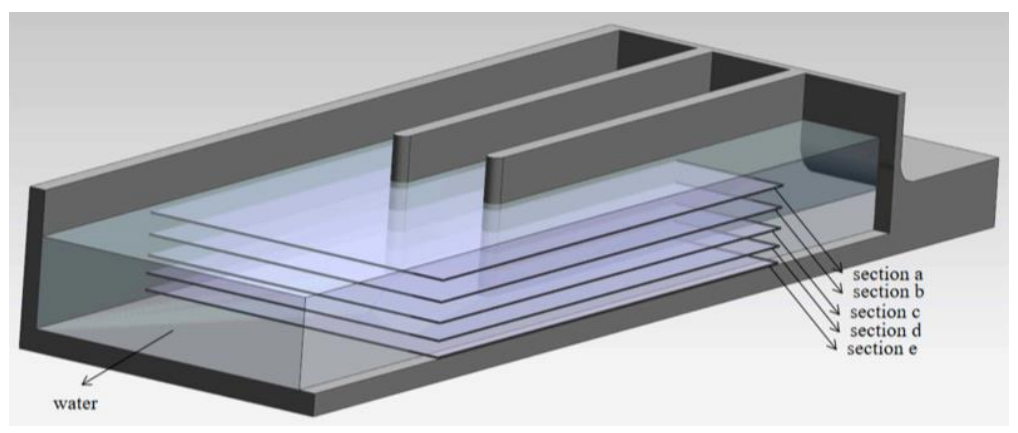

Figure 9: Location diagram of specific sections. 
Different horizontal sections were set up in the test section at the junction of the forebay and pump sump. The PIV camera was installed above the test section, and the laser was placed on the side of the test section to generate a horizontal laser surface. Five flow-layer sections were set at the junction of the forebay and pump sump to study the flow patterns of the non-side and side pump sumps of different flow layers. The specific section locations are shown in Fig. 9.

The distribution of the velocity cloud map of the flow field of different flow layers was obtained from the analysis of experimental data and is shown in Fig. 10, it indicates that the flow velocity distribution of the non-side and side pump sumps was different (Fig. $10 \mathrm{a}-$ Section a). The flow velocity distribution in each water inlet channel of the non-side pump sump was uniform. The side pump sump had a higher flow velocity near the isolated pier, and the flow velocity near the side wall decayed sharply along the flow direction. The downstream side of the side pump sump had a tendency of low-velocity vortex adhering to the wall. As shown in Fig. 10 b, the velocity distribution in the flow field of the side pump sump was similar to that of upper layer a, but the trend of adhering vortices on the downstream side of the inlet side wall increased, and the velocity area at the inlet of the partition decreased. As shown in Figs. $10 \mathrm{c}$ and $10 \mathrm{~d}$, the flow velocity at the downstream side wall of the side pump sump was further reduced, and an adherent vortex was generated near the wall on the downstream. As shown in Fig. 10 e, the flow velocity of the side and non-side pump sumps was further reduced, and the flow of the side pump sump produced an adherent vortex on the downstream side wall. This vortex was caused by the asymmetry of the force on both sides of the water flow when the water entered the inlet. The frictional resistance between the inflow water body and the solid side wall near the side wall was greater than the shear stress between the water flow and the water flow in front of the isolated pier. The results of this analysis based on PIV data are similar to the results of the numerical simulation, indicating that the analysis of the cause of the adherent vortex in the side pump sump is credible.

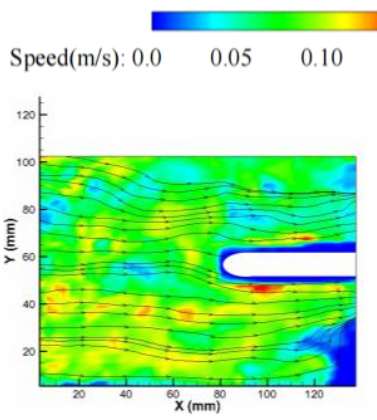

Section a

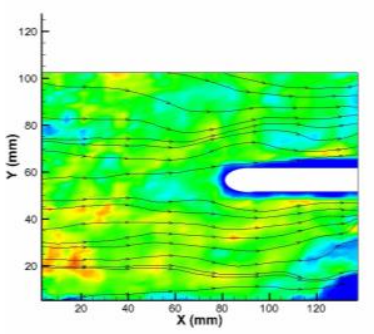

Section d

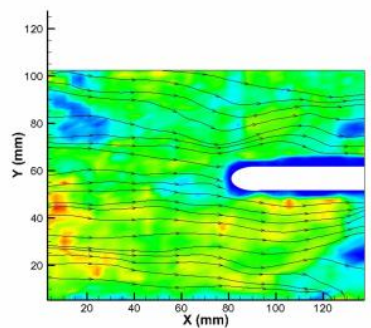

Section $b$

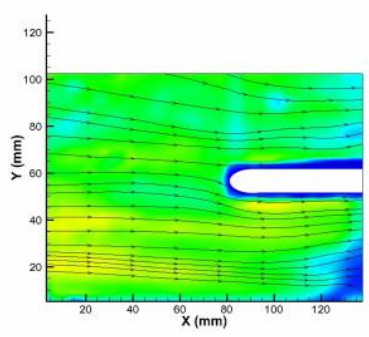

Section e

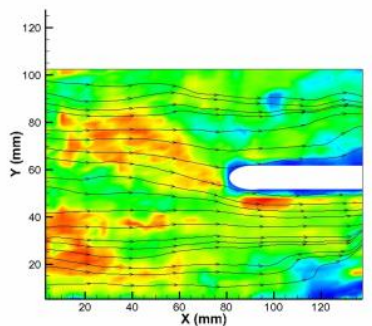

Section c

Figure 10: Flow velocity nephogram of the horizontal plane based on the PIV test.

\section{CONCLUSION}

The vortex problem in the side intake tank of a pumping station affects the station's normal operation and safety. In this study, a numerical model was established to analyse the flow 
field characteristics of the side and non-side pump sumps, and the turbulent kinetic energy equation was used to solve the problem. The conclusions from the numerical model analysis were verified by the data collected from PIV in three aspects: the flow field in different flow layers, the vertical axial section, and the vorticity field. This study revealed that the asymmetry of inflow force in the side pump sump is the fundamental cause of vortexing, and the following general conclusions were obtained.

(1) The force on both sides of the flow before entering the side pump sump is asymmetric. The friction between the water flow that is close to the sidewall and the solid sidewall is greater than the shear stress before the water flows into the isolated pier.

(2) The distribution uniformity of the velocity field differs in the side and non-side pump sumps. The velocity near the sidewall of the side pump sump is less than that near the isolated pier. The flow direction inclines from the entrance to the downstream of the diaphragm isolated pier, forming a low-speed vortex zone near the downstream sidewall. However, no such phenomenon occurs in the non-side pump sump, and the velocity distribution in it is even.

(3) Given that the entrance is below the pumping station, the flow velocity in the pump sump increases gradually from the top to the bottom of the downstream. Therefore, a vertical spiral vortex is easily generated when the water in the side pump sumps flows horizontally into the isolated pier.

Through numerical simulation, theoretical analysis, and a PIV test, this study clarified that the source of vortexes in the side pump sump of a pumping station is the asymmetric inflow force caused by its structural arrangement. This work serves as an important directive for the improvement and control of the flow pattern in the side pump sump. In future research, a vortex elimination method can be determined through further theoretical deduction, experimental research, and field investigation.

\section{ACKNOWLEDGEMENT}

The work was supported by the Jiangsu Graduate Research and Innovation Program (KYCX20_2980), the National Natural Science Foundation of China (No. 51779215; No. 52079120) and the Priority Academic Program Development of Jiangsu Higher Education Institutions (PAPD).

\section{REFERENCES}

[1] Ahmed, F. (2000). Three-dimensional mean velocity analysis of a 30 degree bend flow, Journal of Engineering Mechanics, Vol. 126, No. 12, 1262-1272, doi: $10.1061 /($ asce $) 0733-$ 9399(2000)126:12(1262)

[2] Wang, H. L.; Long, B.; Yang, Y.; Xiao, Y.; Wang, C. (2020). Modelling the influence of inlet angle change on the performance of submersible well pumps, International Journal of Simulation Modelling, Vol. 19, No. 1, 100-111, doi:10.2507/IJSIMM19-1-506

[3] Wang, H.; Qian, Z.; Zhang, D.; Wang, T.; Wang, C. (2020). Numerical study of the normal impinging water jet at different impinging height, based on Wray-Agarwal turbulence model, Energies, Vol. 13, No. 7, Paper 1744, 15 pages, doi:10.3390/en13071744

[4] Wang, C.; Chen, X.; Qiu, N.; Zhu, Y.; Shi, W. (2018). Numerical and experimental study on the pressure fluctuation, vibration, and noise of multistage pump with radial diffuser, Journal of the Brazilian Society of Mechanical Sciences and Engineering, Vol. 40, No. 10, Paper 481, 15 pages, doi: $10.1007 / \mathrm{s} 40430-018-1355-6$

[5] Krantovska, O.; Petrov, M.; Ksonshkevych, L.; Orešković, M.; Synii, S.; Ismailova, N. (2019). Numerical simulation of the stress-strain state of complex-reinforced elements, Technical Journal, Vol. 13, No. 2, 110-115, doi:10.31803/tg-20190417112619

[6] Wang, C.; Shi, W.; Wang, X.; Jiang, X.; Yang, Y.; Li, W.; Zhou, L. (2017). Optimal design of multistage centrifugal pump based on the combined energy loss model and computational fluid dynamics, Applied Energy, Vol. 187, 10-26, doi:10.1016/j.apenergy.2016.11.046 
[7] Zeng, C.; Li, C. W. (2010). A hybrid RANS-LES model for combining flows in open-channel Tjunctions, Journal of Hydrodynamics, Vol. 22, No. 1, 154-159, doi:10.1016/s10016058(09)60186-4

[8] Shin, B. (2018). Effect of submergence and flow rate on free surface vortices in a pump sump, International Journal of Fluid Mechanics Research, Vol. 45, No. 3. 225-236, doi:10.1615/ interjfluidmechres.2018020379

[9] Qian, Z. D.; Wu, P. F.; Guo, Z. W.; Huai, W. X. (2016). Numerical simulation of air entrainment and suppression in pump sump, Science China Technological Sciences, Vol. 59, No. 12, 1847 1855, doi:10.1007/s11431-016-0237-8

[10] Yamade, Y.; Kato, C.; Nagahara, T.; Matsui, J. (2020). Suction vortices in a pump sump-their origin, formation, and dynamics, Journal of Fluids Engineering, Vol. 142, No. 3, Paper 031110, 26 pages, doi: $10.1115 / 1.4045953$

[11] Song, X.; Liu, C. (2019). Experimental investigation of pressure pulsation induced by the floorattached vortex in an axial flow pump, Advances in Mechanical Engineering, Vol. 11, No. 3, 13 pages, doi:10.1177/1687814019838708

[12] Ahmad, Z.; Jain, B.; Kumar, S.; Mittal, M. K. (2011). Rational design of a pump-sump and its model testing, Journal of Pipeline Systems Engineering and Practice, Vol. 2, No. 2, 53-63, doi:10.1061/(asce)ps.1949-1204.0000074

[13] Kim, H.-J.; Park, S. W.; Rhee, D. S. (2017). Numerical analysis of the effects of anti-vortex device height on hydraulic performance of pump sump, KSCE Journal of Civil Engineering, Vol. 21, No. 4, 1484-1492, doi:10.1007/s12205-016-0714-Z

[14] Norizan, T. A.; Reda, E.; Harun, Z. (2018). Enhancement of vorticity reduction by floor splitter in pump sump to improve pump efficiency, Sustainable Energy Technologies and Assessments, Vol. 26, 28-36, doi:10.1016/j.seta.2017.06.001

[15] Hoyas, S.; Jiménez, J. (2008). Reynolds number effects on the Reynolds-stress budgets in turbulent channels, Physics of Fluids, Vol. 20, No. 10, Paper 101511, 8 pages, doi:10.1063/ 1.3005862

[16] Hoyas, S.; Jiménez, J. (2006). Scaling of the velocity fluctuations in turbulent channels up to $R e_{\tau}=2003$, Physics of Fluids, Vol. 18, No. 1, Paper 011702, 4 pages, doi:10.1063/1.2162185

[17] Adrian, R. J. (2007). Hairpin vortex organization in wall turbulence, Physics of Fluids, Vol. 19, No. 4, Paper 041301, 16 pages, doi: $10.1063 / 1.2717527$

[18] Dixit, S. A.; Ramesh, O. N. (2008). Pressure-gradient-dependent logarithmic laws in sink flow turbulent boundary layers, Journal of Fluid Mechanics, Vol. 615, 445-475, doi:10.1017/ s0022112008004047

[19] Abreu, L. I.; Cavalieri, A. V. G.; Schlatter, P.; Vinuesa, R.; Henningson, D. S. (2020). Resolvent modelling of near-wall coherent structures in turbulent channel flow, International Journal of Heat and Fluid Flow, Vol. 85, Paper 108662, 11 pages, doi:10.1016/j.ijheatfluidflow.2020.108662

[20] Albers, M.; Meysonnat, P. S.; Fernex, D.; Semaan, R.; Noack, B. R.; Schröder, W. (2020). Drag reduction and energy saving by spanwise traveling transversal surface waves for flat plate flow, Flow, Turbulence and Combustion, Vol. 105, 125-157, doi:10.1007/s10494-020-00110-8

[21] Yuan, S.; Tang, H.; Xiao, Y.; Qiu, X.; Zhang, H.; Yu, D. (2016). Turbulent flow structure at a 90-degree open channel confluence: Accounting for the distortion of the shear layer, Journal of Hydro-environment Research, Vol. 12, 130-147, doi:10.1016/j.jher.2016.05.006

[22] Song, X.; Huang, C.; Zhang, G.; Shan, W.; Wang, Y. (2019). Experimental study on seismic performance of new-type composite shear wall, DYNA - Ingenieria e Industria, Vol. 94, No. 4, 465-472, doi: 10.6036/9192

[23] Du, X.-X.; Lambert, M. F.; Chen, L.; Hu, E. J.; Xi, W. (2020). Pipe burst detection, localization, and quantification using the transient pressure damping method, Journal of Hydraulic Engineering, Vol. 146, No. 11, Paper 04020077, 14 pages, doi:10.1061/(asce)hy.1943$\underline{7900.0001810}$ 